\title{
Measurement of the velocities in the transient acceleration process using all-fiber photonic Doppler velocimetry
}

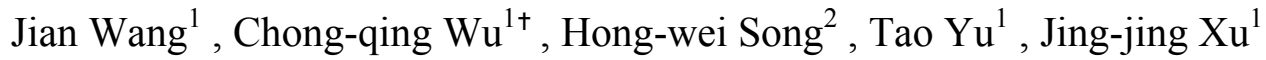 \\ (1. Institute of Optical Information, Key Lab of Education Ministry on Luminescence and Optical \\ Information Technology, Beijing Jiaotong University , Beijing 100044 , China; \\ 2. Key Laboratory for Hydrodynamics and Ocean Engineering, Institute of Mechanics, Chinese \\ Academy of Sciences , Beijing 100190 , China)
}

\begin{abstract}
Based on analysis of basic photonic Doppler velocimetry (PDV), a formula to measure velocity variation in a single cycle is put forward. PDV has been improved in three aspects, namely, the laser, the detector and the data processing. A measurement system for velocity of the initial stage of a shock motion has been demonstrated. Instantaneous velocity measurements have been performed. The experimental results have a good agreement with the values obtained from the accelerometer. Compared with the traditional fringe method, the proposed method in this paper can identify instantaneous velocity variation. So it is particularly suitable for measuring the velocity in the transient acceleration process of shock waves and detonation waves.
\end{abstract}

Optical fiber sensor; Instantaneous velocity mearuement; All-fiber photonic Doppler velocimetry; Shock waves and detonation waves

\section{Introduction}

Laser velocity measurement is one of the primary method for investigating shock waves, detonation waves, et cetera. It is also used in development of new materials, fine processing technology, seismic wave detection and noise control. With the maturity of optical fiber technology and improvement of optoelectronic devices, replacing the traditional velocimeter with all-fiber velocity interferometer has become a trend ${ }^{[1-3]}$. In 2004, O. T. Strand et al proposed a heterodyne velocimetry ${ }^{[4]}$, also called photonic Doppler velocimetry (PDV). Using the square-law detection characteristic of the detector, the beat signal between the signal light and the reference light is generated at the detector. The beat signal is then recorded on a digitizer. The velocity of the moving object is obtained by calculating the number of the beat period (also called interference fringe number). Then they improved the velocimetry and built a four-channel system ${ }^{[5]}$. Subsequently, other researchers made a lot of similar work ${ }^{[6-9]}$, verified the system, and improved the measurement accuracies.

Although the PDV based on the fringe method has many advantages, such as compact structure, low costs, and insensitive to random fluctuations of light intensity, its suitable range is limited. When using fringe method in PDV, the moving distance of the object should be greater than one-half the light wavelength, otherwise, a full beat cycle could not be observed. However, in detonation experiment, the transient acceleration process in the beginning of the movement is even more important. At this stage, the movement time of the object is very short, initial velocity is very small but acceleration is very large. The measured fringe number is less than one or only a few. It is difficult to achieve the precise measurement of transient velocity.

For this reason, a formula to measure accurately velocity in a single cycle is put forward, and PDV has been improved. The instantaneous velocity in the initial stage of a shock motion is obtained by the improved PDV and the formula.

\section{Principle of photonic Doppler velocimetry}

A basic PDV structure is shown in Fig.1. The light from the laser is transported to port 1 of the circulator, then from

† Tel : 01051688046 Email: cqwu@bjtu.edu.cn

21st International Conference on Optical Fiber Sensors, edited by Wojtek J. Bock, Jacques Albert, Xiaoyi Bao, Proc. of SPIE Vol. 7753, 77536F · C 2011 SPIE · CCC code: 0277-786X/11/\$18 · doi: 10.1117/12.886469 
port 2 to the sensing probe. At the probe surface, a part of the light reflected is as the reference light beam; another part transmitted out of the surface is as the signal light beam, which illuminates the moving surface and reflected with Doppler shift back to the probe again. The reference and the signal light beams are sent from port 3 to the detector. Depending on the square-law detection characteristic of the detector, the beat signal is generated at the detector by mixing the two beams and is then recorded on the digitizer.

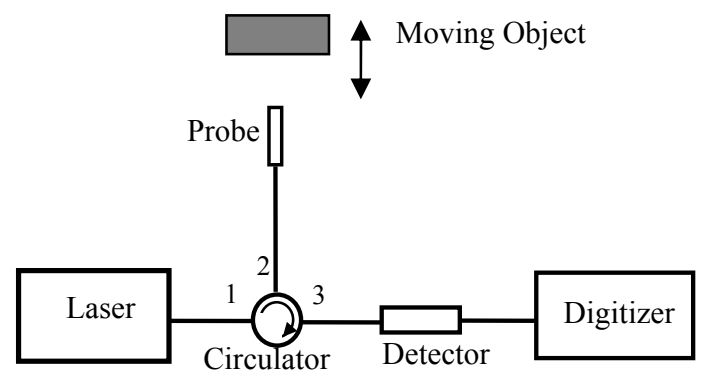

Fig. 1 PDV system principle diagram

Assume the electrical field amplitudes of reference and signal beams are $E_{10}$ and $E_{20}$, respectively, the original frequency of light is $f$, the phases are $\varphi_{1}(t)$ and $\varphi_{2}(t)$. Then the two light fields at the detector are expressed as

$$
E_{1}=E_{10} \cos \left[2 \pi f t+\varphi_{1}(t)\right], \quad E_{2}=E_{20} \cos \left[2 \pi f t+\varphi_{2}(t)\right]
$$

The phase of reference light is a constant independent from the moving object, so $\varphi_{1}(t)=\varphi_{10}$. Due to Doppler Effect, the phase of the signal beam is given by

$$
\varphi_{2}(t)=\varphi_{20}+\frac{4 \pi}{\lambda} \int_{0}^{t} u(t) \mathrm{d} t
$$

where $\varphi_{2}(0)=\varphi_{20}, u(t)$ is the instantaneous velocity of the object. Therefore, the light intensity at the detector can be written as

where $\Delta \varphi=\varphi_{20}-\varphi_{10}$. Assume

$$
I=I_{1}+I_{2}+2 \sqrt{I_{1} I_{2}} \cos \left[\frac{4 \pi}{\lambda} \int_{0}^{t} u(t) \mathrm{d} t+\Delta \varphi_{0}\right]
$$

$$
N=\operatorname{Int}\left[\frac{2}{\lambda} \int_{0}^{t} u(t) \mathrm{d} t\right]
$$

where Int means to get integer, and $N$ is the observed periodic number (the fringe number). The velocity of the moving object is then related to $N$ by

$$
u(t)=\frac{\lambda}{2} \frac{\mathrm{d} N}{\mathrm{~d} t}=\frac{\lambda}{2 \tau(t)}
$$

where $\tau(t)$ is the beat period. It is easy to see that the accurately measured light intensity $I_{1}$ and $I_{2}$ are not required using the fringe method. So the method has the high ability of anti-interference. Besides, to avoid low frequency interference and enhance detection sensitivity, high-speed AC detector usually be used, which has greater than $10 \mathrm{kHz}$ low-cut-off frequency.

Evidently, $u(t)$ in Eq.(5) is the average velocity of a cycle. It is difficult to measure accurately the velocity of the object driven by an impulsive force. To solve the problem, a new formula is derived as follows. From Eq. (3), the maximum and minimum intensity are given by

$$
I_{\max }=I_{1}+I_{2}+2 \sqrt{I_{1} I_{2}}, \quad I_{\min }=I_{1}+I_{2}-2 \sqrt{I_{1} I_{2}}
$$

therefore

$$
I_{1}+I_{2}=\left(I_{\max }+I_{\min }\right) / 2, \quad 2 \sqrt{I_{1} I_{2}}=\left(I_{\max }-I_{\min }\right) / 2
$$

Find the first derivative of Eq. (3) with respect to time, then $u(\mathrm{t})$ is expressed as follows: 


$$
u(t)=-\frac{\lambda}{4 \pi} \frac{\mathrm{d} I}{\mathrm{~d} t}\left\{\sqrt{\left[\left(I_{\max }-I_{\min }\right) / 2\right]^{2}-\left[I(t)-\left(I_{\max }+I_{\min }\right) / 2\right]^{2}}\right\}^{-1}
$$

From this, it can be seen that the absolute value of intensity versus time $I(t)$ should be measured to calculate $u(t)$. In order to measure accurately $I(t)$, the PDV has been improved. Firstly, replace the AC detector with a DC-AC detector which has bandwidth from DC to $10 \mathrm{GHz}$. Secondly, maintain the stability of $I_{\max }$ and $I_{\min }$. For this reason, we design more stable light source and use a DC detector to monitor the source power. The power fluctuations are less than $0.3 \mathrm{~dB}$. Furthermore, the linewidth is as narrow as $200 \mathrm{kHz}$, which provide better fringe contrasts. Thirdly, eliminate noise in the recorded data. Considering the hardware can not suppress the noise in order to obtain the DC and high frequency AC components of the beat signal, the software filtering technique and function fitting are used.

\section{The experimental results and data analysis}

The experimental setup is shown in Figure 2. The semiconductor laser (CQF938/400) produced by JDSU has 100 mw output operating at $1553.29 \mathrm{~nm}$ with linewidth of $209.6 \mathrm{KHz}$. Vibrating object is an aluminum sheet driven by a vibration generator(4089) produced by B\&K. The probe is a GRIN lens. The beat signal is recorded by a LeCroy digitizer with a photodetector.
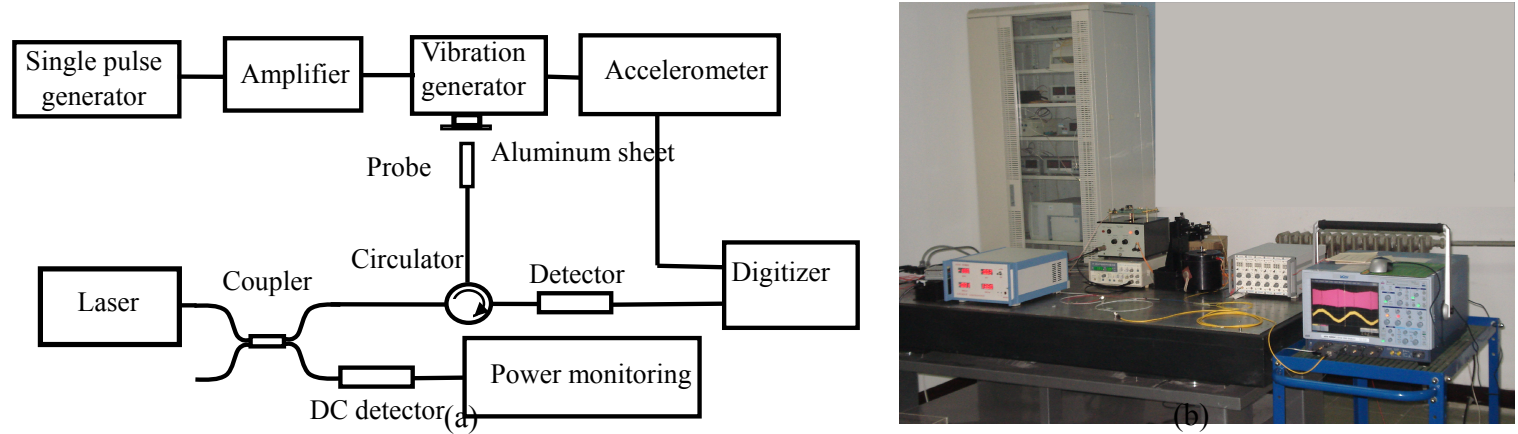

Fig. 2 Experimental system

(a) block diagram (b) picture

To study the transient process of shock motion, we have developed a single pulse generator which can produce a $250 \mu$ s rectangular pulse. The both signal and reference light beams are sent to the detector, and then the beat signal is displayed and recorded by the digitizer. The acceleration of aluminum sheet is measured by the accelerator and is also displayed and recorded by the digitizer. The experimental result for the signal pulse is shown in Fig. 3. The measured beat signal is on the top and acceleration is on the bottom.

From the figure we can see that noise exists in the recorded data. The noise will lead to great errors of differential operation $\mathrm{d} I / \mathrm{d} t$. To solve the problem, we obtain the frequency spectrum of the beat signals using fast Fourier transform, and after removing the noise frequency components we obtain the signal in time domain again using inverse fast Fourier transform. Even so, there is still noise in the beat frequency range which is not easy to eliminate. For this reason, we process the data again using the curve fitting. In the end, the speed and acceleration are calculated using the processed data and Eq.(8), shown in Figure 4 and 5.

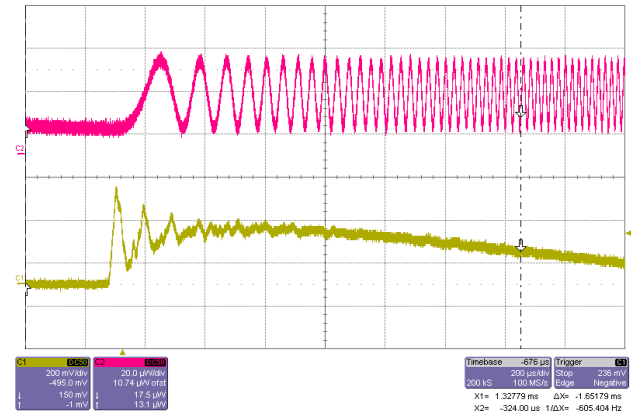

Fig. 3 The interference fringes and the acceleration

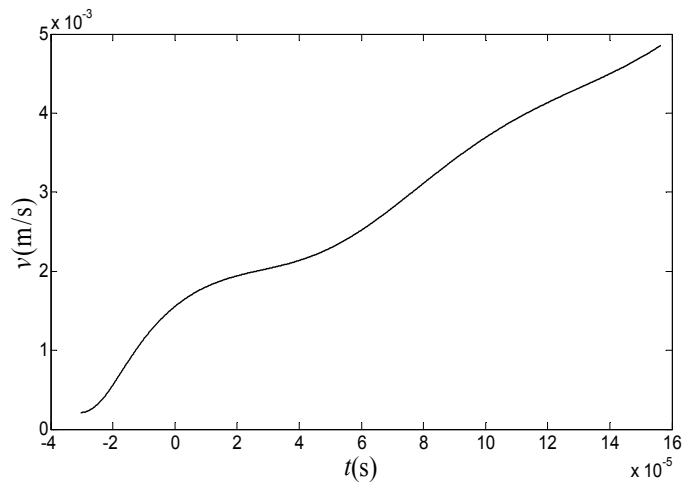

Fig. 4 The velocity of the moving object as a function of the time 
The acceleration curve in Fig. 5 is obtained using PDV. It has a good agreement with the cure on the bottom of Fig.3 which is measured using the accelerator. That shows that our PDV can accurately measure the transient acceleration process.

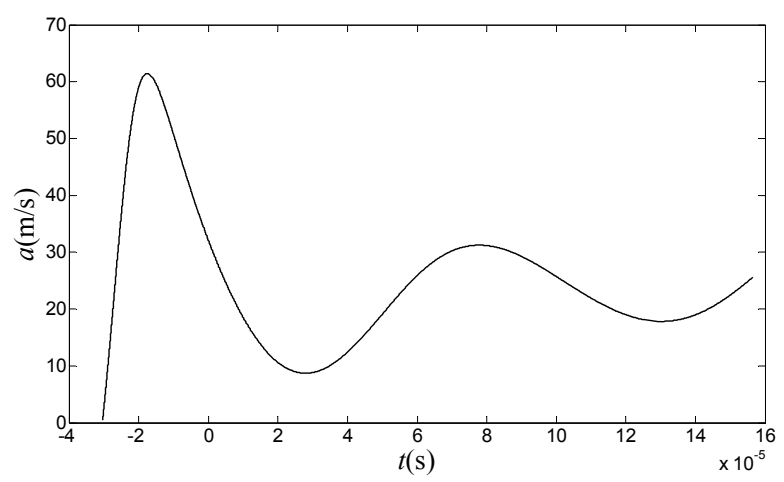

Fig. 5 The acceleration of the moving object as a function of the time

\section{Conclusion}

In this paper, we propose a formula used to measure accurately velocity in a single cycle and improve the basic PDV, namely, using a DC-AC detector instead of the original high-speed AC detector, designing a more stable light source which power fluctuations in the range of $0.3 \mathrm{~dB}$, eliminating noise in the original data using filtering and fitting method. Then we measure instantaneous velocity of a shock motion using PDV and an accelerator. The results measured by PDV are good agreement with that one obtained from the accelerator. This shows that it is correct to calculate instantaneous velocity using Eq. (8) and this method is particularly suitable for measuring transient acceleration process of the explosive wave and shock wave.

\section{Acknowledgments}

This research is supported by the National Natural Science Foundation of China (grant 60877057 and 60907027).

\section{References}

[1] Levin L , Tzach D., "Fiber Optic velocity interferometer with very short coherence length light source," Rev Sci Instrum, 67(4), 1434-1437 (1996)

[2] Fabiny, L., Kersey A. D., "Interferometic Fiber-Optic Doppler Velocimeter with High-Dynamic Range," IEEE PTL, 9(1), 79-81 (1997)

[3] Weng J. D., Tan H., Cneng J. B., et al., "Application of Fiber Velocity Interferometer System for Any Reflector in High Pressure Physics," Chinese Journal of High Pressure Physics, 18(3), 225-230 (2004)

[4] Strand O.T.and Birzins L.V., "Velocimetry using heterodyne techniquie," UCRL-CONF-206034, 2004

[5] Strand O.T., Goosman D. R., Martinez C., et al., "Compact system for high-speed velocimetry using heterodyne teehniques," Rev Sci Instrum, 77, 083108, (2006)

[6] Jensen B. J., Holtkamp D. B., Rigg PA, et al., "Accuracy limits and window corrections for photon Doppler velocimetry," J. Appl. Phys., 101, 013523 (2007)

[7] Dlan D. H.and Jones S. C., "Push-pull analysis of photonic Doppler velocimetry measurements," Rev Sci Instrum, 78, $076102(2007)$

[8] Mercier P., Bénier J., Frugier P.A., et al., "Heterodyne velocimetry and detonics experiments," Proc. of SPIE, 7126, 71261O-1 (2009)

[9] Wang D. T., Li Z. R., Wu J. R., et al., "An Optical-fiber Displacement Interferometer for Measuring Velocities of Explosviey-driven Mental Plates," Explosion and Shock Waves, 29(1), 105-108 (2009) 